\title{
Induction in Adult Acute Lymphoblastic Leukaemia: A Study of 22 Cases
}

\author{
H KHATUN $^{\mathrm{a}}$, M MORSHED ${ }^{\mathrm{b}}$, S ANAM ${ }^{\mathrm{c}}$
}

\begin{abstract}
Summary:
Treatment of acute lymphoblastic leukaemia yields high complete remission (CR) rate with disappointingly low overall survival. We describe the results of induction in 22 cases of adult acute lymphoblastic leukaemia treated between January 2011 and January 2013. Median age at presentation was 28 years (range 13-70). Fatigue ( $n=15,68 \%)$ was the most frequent presenting complaint followed by fever $(n=14$, $63.7 \%) .36 .3 \%$ patients presented with splenomegaly while $31.8 \%$ had lymphadenopathy. The average presenting white cell count was $61 \mathrm{k} / \mathrm{ul}$. B-ALL was more common than TALL (59\% and 27\% respectively). 8 cases were deemed high risk and 14 cases were standard risk. One patient died early in induction before specific treatment. 20 patients were treated with intensive chemotherapy (CALGB8811=12, MCP 841=4,
\end{abstract}

Introduction:

Acute lymphoblastic leukaemia (ALL) is a malignancy of lymphoblasts involving blood and bone marrow (BM):the diagnostic cut off is e" $25 \%$ lymphoblasts in $\mathrm{BM}^{1}$. The onset is usually acute with symptoms of few weeks duration. Up to one half of the patients have documented infection and one third has bleeding manifestation as presenting features ${ }^{2}$. The diagnostic work up for ALL now includes examination of blood and bone marrow films, flowcytometry, cytogenetic and molecular genetic analysis. Established prognostic factors include age, high white cell count at presentation (>30 k/ul for B-ALL,>100 k/ul for T-ALL), abnormal cytogenetics including $t(9 ; 22)$ and $t(4 ; 11)$, steroid response, time to complete remission (CR) ( $>4$ weeks ) and persistent minimal residual disease $(\mathrm{MRD})>10^{-4}$ for 3-4 months. Some ALL treatment groups include pro-B, early $\mathrm{T}$ and mature $\mathrm{T}$ phenotypes, $\mathrm{t}(1 ; 19)$ and

a. Dr. Hazera Khatun, Specialist, Haematology, Square Hospital.

b. Prof. Manzur Morshed, Consultant, Haematology, Square Hospital.

c. Dr. Sadia Anam, Resident Medical Officer, Haematology, Square Hospital.

Address of Correspondence: Dr. Hazera Khatun, Specialist, Haematology, Square Hospital, Tel: 88-01923745775, E-mail: drhazerak56@gmail.com hazera.khatun@squarehospital.com

Received: 2 November, 2013

Accepted: 20 October, 2014
$C C G-B F M=2$ and $B F M-90=1$, others 1). Overall CR was 85\% while CR in CALGB8811 was 83.3\%.The average time to NADIR was 10 days (range 6 -17 days). The most common complication in induction was neutropenic sepsis $(n=19$, $86.4 \%$ ) contributing alone to induction mortality of $15 \%$ $(n=3)$. Causative organism was identified in only $31.8 \%$ cases $(n=7)$, the commonest being pseudomonas and coagulase negative staphylococcus. Facilities for extended immunophenotyping and cytogenetic tests (in addition to $B C R-A B L$ by $P C R$ ) are required for administration of standard of treatment.

Key words: Acute lymphoblastic leukaemia, Induction chemotherapy.

(J Banagladesh Coll Phys Surg 2015; 33: 18-22)

complex karyotype abnormalities for further stratification ${ }^{3,4}$.

Treatment of ALL is divided into induction, CNS targeted therapy, consolidation therapy and a prolonged maintenance ${ }^{4}$. Induction consists of multi agent chemotherapy with vincristine and prednisolone as backbone. Other agents used are cyclophosphamide, anthracycline, L-asparaginase. Consolidation can be with chemotherapy or stem cell transplantation depending on risk status, availability of donor and facilities for stem cell transplantation ${ }^{3}$. Adult ALL treatment has been inspired and guided by treatment response in pediatric ALL. The Berlin-FrankfurtMunster (BFM) group pioneered successful multi-agent pediatric ALL treatment in $1980^{5,6}$. The Cancer and Leukaemia Group B (CALGB 8811) uses a 5 drug induction and schedules Daunoribucin (dauno) in 3 consecutive days. This protocol uses L-asparaginase (Lasp) and achieved $86 \%$ CR with 3 year DFS of $46 \%$ and 3 year OS of $50 \%$ only. Intensification of the dauno without the use of L-asp in induction and subsequent high dose Methotrexate and Ara-c consolidation (CALGB 19802) improved CR(92\%) without significant improvement of OS $(47.7 \% \text { only })^{6}$. UKALLXII/ECOG2993 trial yielded 91\% CR with 5 year OS of $41 \%$ in younger adults[7].The UKALLXII, however, uses dauno as $60 \mathrm{mg} / \mathrm{mt}^{2}$ on weekly basis. 
Kantiranjan successfully used vincristine, adriamycin and dexamethasone for adult ALL induction which later resulted in exclusion of $\mathrm{L}$-asp in hyper-CVAD regimen ${ }^{8,9}$.

Economic constraints and limitation of diagnostic facility impede administration of standard of management to acute leukemia patients in Bangladesh and adult ALL is no exception. Inadequacy of supportive care, infection control and growth factor support hinder the administration of dose intense protocol like hyperCVAD. There is no consensus on ALL treatment protocols among the practicing physicians in Bangladesh. Scarcity of systematic data collection and analysis makes the situation worse. We describe the outcome of induction in adult ALL patients to determine the practicalities of treatment in order to identify scope of improvement and decide on a protocol suitable for our country.

\section{Materials and methods:}

Adult (over 12 years) ALL patients were treated over a period of January 2011 to March 2013. Both male and female patients were included. Diagnosis was confirmed by peripheral blood and bone marrow morphology aided by flowcytometry. RT-PCR for BCR-ABL was done in all B-ALL patients at the minimum. Risk status at presentation was determined on the basis of age, presenting count, immunophenotype and cytogenetics. Packed red cells were transfused when $\mathrm{Hb}<8 \mathrm{~g} / \mathrm{dL}$, platelet transfusion was given prophylactically at platelet count $5 \mathrm{k} / \mathrm{ul}$ or less and in presence of life threatening bleeding. Blood counts were repeated every day during the initial critical period and every alternate day thereafter. Response to treatment was assessed by bone marrow (BM) aspiration on D28 of induction. Patients who achieved CR after induction proceeded to early intensification, CNS prophylaxis, late intensification and maintenance as per protocols they were enrolled in. Patient's data are used anonymously and after obtaining informed consent. Data analysis was done using MS Excel.

\section{Results:}

\section{Patient characteristics:}

A total of 22 patients of adult ALL were treated over a period of January 2010 to March 30, 2013. Median age of presentation was 28 years (range 13 to 70 years).
Majority of the patient belonged to the age group 15$25(n=8,36.3 \%)$ followed by $26-35(n=6,27.2 \%) .15$ patients were male and 7 female. $68 \%$ of the patients presented with fever and fatigue. About one third patients $(n=8)$ had haemorrhage; severity of WHO I in 5 , WHO II in 2 patients and WHO III/IV in one patient only. 36\% patients had splenomegaly at presentation while $31.8 \%$ had lymphadenopathy and bony tenderness (table I).One patient had headache and drowsiness with bilateral squint at presentation which was later confirmed by CT brain to be due to multiple haemorrhagic spots in cerebral hemispheres and brain stem.

\section{Table-I}

\begin{tabular}{|c|c|c|}
\hline \multicolumn{3}{|c|}{ Baseline clinical characteristics } \\
\hline Presenting feature & Number of patients & $\%$ \\
\hline \multicolumn{3}{|l|}{ Age } \\
\hline median & 28 & \\
\hline range & 13 to 70 & \\
\hline \multicolumn{3}{|l|}{ Gender } \\
\hline Male & 15 & 68.18 \\
\hline Female & 7 & 31.82 \\
\hline Fever & 14 & 63.64 \\
\hline Fatigue & 15 & 68.18 \\
\hline Bleeding & 8 & 31.82 \\
\hline WHO I & 5 & \\
\hline WHOII & 2 & \\
\hline WHO III & 1 & \\
\hline Headache & 1 & \\
\hline Splenomegaly & 8 & 36.36 \\
\hline Hepatomegaly & 1 & 4.55 \\
\hline Lymphadenopathy & 7 & 31.82 \\
\hline Bony tenderness & 7 & 31.82 \\
\hline
\end{tabular}

\section{Laboratory features:}

The average presenting white cell count was $65.5 \mathrm{k} / \mathrm{ul}$ : the lowest $1.5 \mathrm{k} / \mathrm{ul}$ and the highest $587.9 \mathrm{k} / \mathrm{ul}$ in a case of T-ALL. Presenting WBC was $>30 \mathrm{k} / \mathrm{L}$ in $40 \%$ of the B-ALL cases and the highest count was $103 \mathrm{k} / \mathrm{ul}$. 33\% patients with T-ALL presented with high WBC >100 k/ ul. About 55\% of the patients had mild to moderate anaemia at presentation and only $18 \%$ were severely anaemic with the lowest $\mathrm{Hb}$ of $3.3 \mathrm{~g} / \mathrm{dL}$ Average $\mathrm{Hb}$ at presentation was Hb $9.0 \mathrm{~g} / \mathrm{dL}$. The average PLT count was $40.9 \mathrm{k} / \mathrm{uL}$ (table II). Immunophenotyping was not 
available in 3 cases. Of the remaining 19, 13 cases were phenotypically B and 6 cases were T-ALL (Fig.1). CD 79a was expressed in all cases of B-ALL followed by CD 19 in 11 cases and CD 10 in 9 cases. Aberrant positivity of CD13 was noted in 2 cases of B-ALL. Phenotypically 10 (77\%) cases were common-ALL and only 3 cases were pro-B ALL (23\%) (Fig.1). 8 cases (36\%) were deemed high risk and 14 cases were standard risk.None of the patients in this series was BCR-ABL positive.

Table-II

\section{Laboratory features at presentation}

\begin{tabular}{l} 
Lab features at presentation \\
$\begin{array}{c}\text { value/ number } \\
\text { of patients } \quad \text { Percentage }\end{array}$ \\
\hline
\end{tabular}

Average $\mathrm{Hb} 9.0 \mathrm{~g} / \mathrm{dL}$

18.18

$\begin{array}{lll}\text { Severe anaemia }(\mathrm{Hb}<6 \mathrm{~g} / \mathrm{dL}) & 4 & 18.18 \\ \text { Moderate anaemia }(\mathrm{Hb} 6-10 \mathrm{~g} / \mathrm{dL}) & 8 & 36.36\end{array}$

Mild anaemia (Hb 10-12 g/dL) $\quad 10 \quad 45.45$

Presenting TWBC $(\mathrm{k} / \mathrm{uL})$

Average

Highest

65.49

Lowest

1.5

B-ALL higest WBC

T-ALL highest WBC

Leukocytosis in B-ALL

(WBC >30 k/ul)

Leukocytosis in T-ALL

( WBC >100k/ul)

PLT(k/ul)

Average

$<10(\mathrm{k} / \mathrm{ul})$

$10-20(\mathrm{k} / \mathrm{ul})$

$>20(\mathrm{k} / \mathrm{ul})$

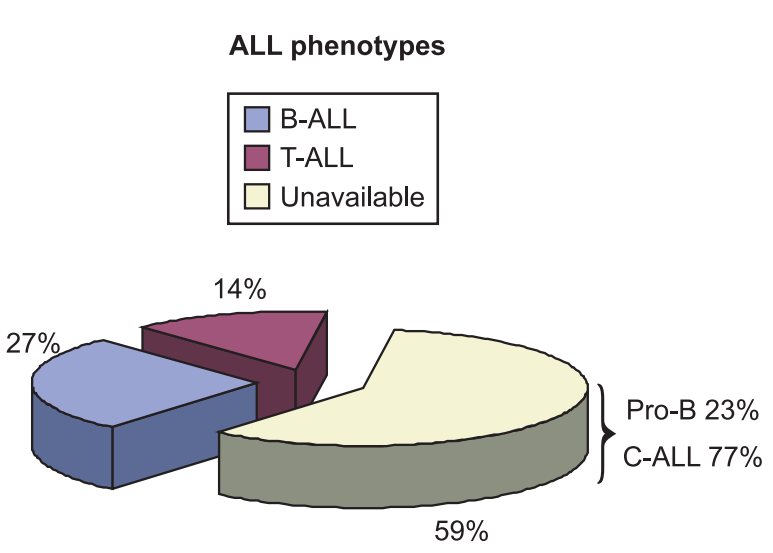

Fig.-1: Immunophenotypic classification of ALL. 59\% of the patients were B-ALL. Of them $23 \%$ of the patients had pro-B phenotype and the rest were common $A L L$ (C-ALL) in phenotype(CD 10+).

\section{Treatment outcome and complications:}

One patient was offered palliative treatment with VCR and Prednisolone considering his age and economic status. One patient died of bilateral pneumonia as complication of the disease before starting induction treatment. The rest were treated with curative intent using intensive chemotherapy (CALGB8811=12 patients, MCP $841=4$ patients and CCG-BFM=2 patients.) Overall CR was $85 \%$ and CR in CALGB8811 was $83.33 \%$. CR in B-ALL was $84.61 \%$ and $80.00 \%$ (Fig.2B). The average time to NADIR was 10 days (range 6 -17 days). Average time to clear blast after steroid treatment was 6.16 days (earliest 3 days and latest 8 days).

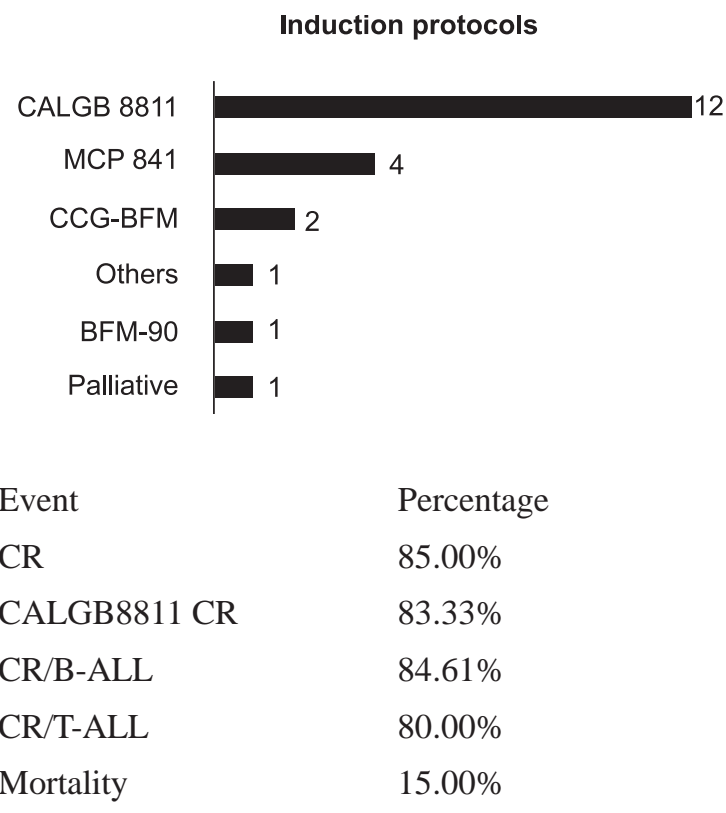

Fig.-2: (a)- Protocols used in induction for ALL and (b)- outcome of induction.

Remission status was assessed by bone marrow morphology on D28. One patient had prolonged neutropenia and BM aspiration was delayed by 2 weeks. The most common complication of treatment was neutropenic sepsis $(n=19,86.36 \%)$ followed by haemorrhage $(n=6,27.27 \%)$ and tumour lysis syndrome $(n=2,9 \%)$. Only one patient developed intracranial haemorrhage which gradually resolved after platelet transfusion. Other complications noted were pancreatitis, hepatitis and thrombosis. Pancreatitis and thrombosis were complications of L-asparaginase. A causative organism was identified in $31.82 \%$ cases. The 
most frequent agents were pseudomonas and coagulase negative staphylococcus ( $\mathrm{n}=2,28 \%$ ) multi-drug resistant Staph. aureaus (MRSA) and E.coli. Reactivation of herpes was observed in one patient.

\section{Discussion:}

The median age of presentation in this study was 28 years ( range 13 to 70 ) which is not different from GIMEMA 0288 (28 years) and LALA 94 (33 years) ${ }^{10,11}$. Up to two thirds of the patients had fever and fatigue at presentation which is higher than in collaborative trials reported by GIMEMA and GMAL ${ }^{10}$. Haemorrhage was the clinical presentation in $31.82 \%$ patients and only 1 had severe haemorrhage (WHO III). 36.36\% patients had splenomegaly at presentation while $31.82 \%$ had lymphadenopathy. Bone pain and joint pain, although described to be more common in children, about 31\% patients in our study had these complaints. Lymphadenopathy was found to be more common in B-ALL than in T-ALL (85\% and 15\% respectively). This could be due to the small number of patients in this study. Hyperleukocytosis was more common in B-ALL (40\%vs 33\% in T-ALL).Majority of the patients had mild to moderate anaemia and only $18 \%$ were severely anaemic at the time of diagnosis which again is similar to the co-operative studies with large number of patients ${ }^{10}$. CNS manifestation was seen in $1-7 \%$ of the patients in CALGB and GIMEMA studies, however, it was absent in our study. Phenotypically, 59\% were BALL and $27 \%$ were T-ALL. B-ALL incidence was lower than reported by Riley et $\mathrm{al}^{12}$. Whether the $14 \%$ of undetermined phenotype patients could compensate this difference is difficult to comment. None of our patients had BCR-ABL rearrangements although it is reported that such rearrangements are found in $20-30 \%$ of adults ${ }^{13}$.In the current study, PCR for BCR-ABL included only the probe for BCR-ABL-210 which allows for failure of detecting BCR-ABL-190 present in about two thirds of the Ph+ ALL patients. However, since majority of the patients (85\%) went into CR without the use of TKI in induction, it can be safely concluded that those patients were truly negative for BCR-ABL. The overall CR rate of $85 \%$ is lower than 92\% CR reported by Hahn and Sive ${ }^{6,7}$. CR by CALGB8811 (83.3\%) was lower than MRCUKALLXII CR. Induction mortality was higher (15\% overall and CALGB8811 $16.6 \%$ ). This is attributable to very small number of patients $^{14}$. Neutropenic sepsis was the most frequent induction complication of which only $31.8 \%$ cases yielded organism in culture. This is consistent with the report from trial of empirical antibiotic therapy performed by the EORTC- IATG. Pseudomonas and coagulase negative staphylococcus were the most frequent organism in neutropenic sepsis. The same study reported shift of gram positive sepsis towards the previously prevalent gram negative sepsis in febrile neutropenia associated with cancer patients ${ }^{15}$.

\section{Long term follow up:}

Until March, 2013 long term follow up was available for 12 patients only. Of these 7 patients are continuing chemotherapy with CALGB8811 protocol and 2 with CCG-BFM. 2 patients are continuing chemotherapy at other haematology institutes. 1 patient continued intensification for 2 cycles but later was switched to palliative treatment considering his co-morbidities and complications of steroid. At median follow up of 8 months (range 3 months to 17 months) 11 patients are alive in remission and 1 patient alive on palliation. Median DFS is 4 months (range 2 months to 16 months).

\section{Conclusion:}

The overall CR in induction and mortality rate is comparable to co-operative group studies on ALL despite the limitation of resources, both diagnostic and therapeutic, in a developing country. There is no alternative to building advanced diagnostics through collaboration of clinicians and molecular biologist in order to achieve the long term goal of delivering standard of care in ALL patients. This study will be continued to test the suitability of CALGB8811 as a dose dense, effective yet practicable protocol for ALL in our setting.

\section{References:}

1. Brunning RD, et al., eds. Precursor B lymphoblastic leukaemia/lymphoblastic lymphoma. 4th ed. In: WHO Classification of Tumours of Haematopoietic and Lymphoid Tissues., ed. Swerdlow SH, et al. 2008, AIRC: Lyon. 91-94.

2. Itakura $\mathrm{H}$ and Coutre SE, eds. Acute lymphoblastic leukemia in adults. 12th ed. In: Wintrobe's Clinical Haematology, ed. F. Greer JP, J, Rodgers GM, Praskevas F, Glader B, Arber DA, Means RT Jr. Vol. 1. 2009, Lippincott Williams and WIlkins: Philadelphia. 1821-1842.

3. Gokbuget N and Hoelzer D, eds. Adult acute lymphoblastic leukaemia. 6th ed. In: Postgraduate Haematology, ed. Hoffbrand AV, et al. 2011, Willey Blackwell: Sussex. 433-447. 
4. Stock W, Adolescents and Young Adults with Acute Lymphoblastic Leukemia. Hematology, 2010: 21-29.

5. Smith MA, Seibel NL, and Altekruse SF et al, Outcomes for children and adolescents with cancer: challenges for the twentyfirst century. . J Clin Oncol., 2010; 28: 2625-2634.

6. Han AR, et al., Outcomes of a Modified CALGB 19802 Regimen in Adult Acute Lymphoblastic Leukemia. J Korean Med Sci, 2008; 23(2): 278-283.

7. Sive JI, et al., Outcomes in older adults with acute lymphoblastic leukaemia (ALL): results from the international MRC UKALL XII/ECOG2993 trial. BJH, 2012; 157: 463-471.

8. Kantarjian H, Thomas D, and O’Brien S et al, Long-term follow up results of hyperfractionated cyclophosphamide, vincristine, doxorubicin, and dexamethasone (hyper-CVAD), a dose-intensive regimen, in adult acute lymphocytic leukemia. Cancer, 2004; 101: 2788-27801.

9. Katarjian H, Walters RS, and Keating MJ et al, Results of the vincristine, doxorubicin, and dexamethasone regimen in adults with standard- and high-risk acute lymphocytic leukemia. . J Clin Oncol., 1990; 8: 994-1004.

10. Annino L, Vegna ML, and Camera A et al, Treatment od adult acute lymphoblastic leukaemia (ALL): Long term follow up of the GIMEMA ALL 0288 randomized study. Blood, 2002; 99: 863-871.

11. Thomas X, B.J., Huguet F et al,, Outcome of treatment in adults with acute lymphoblastic leukaemia: analysis of the LALA-94 trial. Journal of Clinical Oncology, 2004; 22: 4075-86.

12. Riley RS, Masssey D, and Jackson-Cook C et al, Immunophenotypic analysis of Acute lymphocytic leukaemia. Hematol Oncol North Am, 2002; 16: 245-299.

13. Gleissner B, Gokbuget N, and Bartram CR et al, Leading prognostic relevance of the BCR-ABL translocation in adult acute B-lineage lymphoblastic leukaemia: a prospective study by German Multicenter trial group and confirmed by polymerase chain reaction analysis. Blood, 2002; 99: 1536-1543.

14. Rowe JM, et al., Induction therapy for adults with acute lymphoblastic leukemia: results of more than 1500 patients from the international ALL trail: MRAC UKALL XII/ECOG E2993. Blood, 2005; 106(12): p. 3760-67.

15. Viscoli C, V. O, and and Machetti M, Infections in Patients with Febrile Neutropenia:Epidemiology, Microbiology, and Risk Stratification. Clinical Infectious Diseases, 2005; 40: $240-5$. 